\title{
Precision Handling of Electronic Components for PCB Rework
}

\author{
Gianmauro Fontana ${ }^{1}$, Serena Ruggeri ${ }^{1}$, Giovanni Legnani ${ }^{2}$, and Irene Fassi ${ }^{1}$ \\ ${ }^{1}$ Institute of Industrial Technologies and Automation, CNR, Milan, Italy \\ \{gianmauro.fontana, serena.ruggeri, irene.fassi\} aitia.cnr.it \\ ${ }^{2}$ Department of Mechanical and Industrial Engineering, University of Brescia, Brescia, Italy \\ \{giovanni.legnani\}@ing.unibs.it
}

\begin{abstract}
The paper focuses on the study of strategies and tools to handle miniaturized components in the electronic industry. In particular, the paper presents an innovative device and method to manipulate microcomponents by vacuum. The device includes an original releasing system, that does not require any external actuation, to assist their release. Indeed, at the microscale, adhesion forces predominate over the gravitational force due to the small masses of the microcomponents, often leading to the failure of the release phase if a release strategy is not implemented. The device, able to eliminate the adhesion problem, is compared with a traditional vacuum microgripper in terms of grasping and releasing error and percentage. The results of preliminary experimental tests are discussed, demonstrating that the innovative microgripper represents an interesting solution for handling electronic components as well as different microparts.
\end{abstract}

Keywords: Micro-handling, Micro-Robotics.

\section{Introduction}

In the last decades, a number of mechatronic devices have been developed for different purposes and have been broadening more and more in various fields. They result from a suitable integration of mechanical, information technology and electronic features that allow both to enhance the capabilities and the performance of standard products and to enable the conception and development of new generation systems facing the high-demanding requirements of an increasing market.

Products of daily usage such as cars, mobile phones and computers for example rely on electronics and contain Printed Circuit Boards (PCBs). They can mount few or hundreds components such as ball grid array (BGA) packages for integrated circuits, resistances or capacitors, often of tiny size. Indeed, a heavy trend to miniaturization has recently appeared, reflecting the need of getting smaller final products with integrated functionalities.

Despite the manufacturing technologies for electronic components and PCBs appear consolidated, few attention has been paid in the years to the development of suitable techniques to repair, reuse or recycle this kind of products when a malfunctioning or a breakdown occur. Most of times, due to economic reasons, the PCB is substituted with a new one and disposed, wasting many parts that could still have value and be recovered. 
However, it has been estimated that, from an environmental point of view, remanufacturing is $80 \%$ more energy efficient than traditional manufacturing and, from an economic point of view, $60 \%$ more cost efficient [1].

If a repair plan is expected, the rework of PCBs is often performed manually, or in few cases with highly dedicated machines, and can result in a very expensive and complex operation; in the same way, the recovering of components in case of irreparable PCBs is not efficient.

According to the emerging "De-manufacturing" paradigm, innovative approaches and methodologies should be implemented to improve the management of electronic products at their end of life [2].

Consider for example the recovery of a working BGA from a defective PCB. In this case, the BGA is de-soldered from the PCB, by heating and consequently melting the soldering balls (with diameter $<1 \mathrm{~mm}$ ). Then, a procedure called "re-balling" is needed to prepare the chip for the re-use, consisting in attaching new solder balls on the bottom surface of the BGA support. Nowadays, this operation is performed mainly manually with the help of fixtures and stencils or using preforms [3]. Specific stencils or preforms have to be used for each particular BGA support type, then limiting the flexibility of the process, and the operator ability influences the accuracy of the result. Recently, automatic rework stations have been developed [3], but still there is a need for more flexible and cost efficient automated equipment.

In this context, the present paper focuses on the study of methods and tools for handling miniaturized components to move towards a flexible and efficient rework of PCBs. The study is devoted to two main related aspects: the re-balling of BGA packages and the replacement of different surface-mount components (SMCs) on the PCBs. To overcome the limits of the current procedures, the work addressed the development of simple and low-cost gripping devices and strategies for both a complete or selective re-balling, then able to pick and place a whole grid of small solder balls or a single ball precisely and reliably. In the same way, the tools should be able to accomplish the replacement of SMCs of different size and mounted on the PCB with a generic orientation.

In particular, an innovative device and method to manipulate by vacuum a component has been conceived and prototyped. A patent application has been filed in March 2013 [4]. This device has been compared with a conventional vacuum microgripper in the execution of pick and place tests of both solder balls of various diameter and resistances.

The investigation of their performance considered both the gripping and the release phases. Indeed, at the microscale, due to the predominance of adhesion forces (e.g. capillary, electrostatic and Van der Waals forces) over the gravitational force, the release of microcomponents is often prevented or uncertain. In literature many solutions have been proposed to cope with this issue [5], but the search of effective and efficient methods is still an undergoing study. For this reason, a special releasing system has been designed and integrated in the innovative gripping device to release the components precisely, reliably and safely, avoiding an excessive increase in weight of the device itself or complexity of its structure.

The two types of handling devices are presented in Section 2. Section 3 describes the experimental tests and the performance indices considered for the comparison and discuss the preliminary results, highlighting the benefit of the proposed solution. 


\section{The Handling Devices}

A convenient solution for the manipulation of electronic components is represented by the use of vacuum grippers, commonly used for macrocomponents and here adapted to the tiny size of the microcomponents. They are based on the pressure difference between the gripper and the atmosphere and have a generally simple structure, since they are mainly based on a small suction hose; they are not expensive and can be used to manipulate a wide range of components, even fragile.

In this work, two types of vacuum microgrippers were considered. The former is a standard microgripper: it is a commercially available needle for dispensing, basically consisting of a cannula connected to a vacuum generation system. The cannula has an internal diameter of $260 \mu \mathrm{m}$, which is attached to the hollow needle body that can be connected to the end-effector of a robot through a proper mechanical interface.

However, in [6], it has been shown that the release is greatly affected by the presence of the adhesion forces and that the simple switch off of the vacuum is often not sufficient. Therefore, suitable expedients need to be found. For example, the adhesion due to the electrostatic force can be reduced coating the glass pipette for the suction with a conductive layer connected to the ground. Moreover, the microcomponent could be guided to hit a sharp edge or rolled on the release plane. The inertial force of the component, generated by moving the gripper brusquely upwards for a short stroke, can also be exploit to ease the release, although results can be inaccurate [6]. Finally, the application of positive pressure, that is of a soft blow for some milliseconds, allows the achievement of a high release percentage and does not affect negatively the final precision of positioning. However, the tuning of the parameters related to the blow (e.g. its intensity) could sometimes be difficult and a trade-off between percentage and precision of release of the specific component has to be found.

On the basis of the results obtained in past experiments and the study of the detected behaviours, a new vacuum micro-gripping tool (No. 1 in Fig. 1) has been conceived and developed, able to cope with the micropart release issues. Similarly to a standard vacuum microgripper, this device is based on the pressure difference between the gripper and the atmosphere and basically consists of a cannula or suction hose connected to a vacuum generation system for picking a component by suction on the gripping end. It integrates an innovative mechanical system to assist the release phase of micro components precisely, reliably and safely, avoiding a considerable increase in weight of the device itself or an excessive complication of the system. In details, the mechanical system is inserted at least partially in the manipulation device and movable from a release position where a release portion projects externally from the gripping end, and a gripping position where the release portion returns into the manipulation device. The mechanical system includes a transversal extension, that in the current case is a holed disc, and a needle attached to the disc and having a diameter smaller than the internal diameter of the cannula. The needle is inserted at least partially inside the cannula and includes the release portion.

The mechanical release system is designed to be moved from the release position to the gripping position through activation of the vacuum generating system, which sucks in the mechanical release system in opposition to a return force pulling it toward the release position, for example the force of gravity acting on the mechanical release system. It is also designed to be moved from the gripping position to the 
release position by the said return force following a reduction or elimination of the pressure difference between the inside and outside of the manipulation device operated by the vacuum generating system.

A main feature consists in using the actuation principle for the part picking to move the release system, without the need of additional actuators, that would make the system more complex, heavier, bigger and more expensive.

\section{Preliminary Experiments and Results}

In this section, the results obtained through a set of preliminary experiments are presented. The tests evaluated the performance of the two types of grippers both in gripping and releasing different components. In particular, the handling of solder balls with diameter of 500 microns and mass of $0.00055 \mathrm{~g}$, and SMD resistances with size $1.5 \mathrm{x}$ $0.8 \times 0.45 \mathrm{~mm}$ and mass of $0.002 \mathrm{~g}$ was evaluated. Each test consisted in the execution of a set of 30 standard pick and place cycles, representative of the real trajectory that the gripper with the component should perform for assembly or PCB rework.

The tests were carried out exploiting the setup available in our micro-manipulation work-cell [7]: a Mitsubishi Electric RP-1AH robot (Fig. 1) was used as motion system, while the measurements of the position and orientation of the microparts was obtained by a suitable vision system consisting of a camera combined with a macro lens, providing a bottom view of working (gripping and releasing) area with a spatial resolution of about $8.1 \mu \mathrm{m}$.

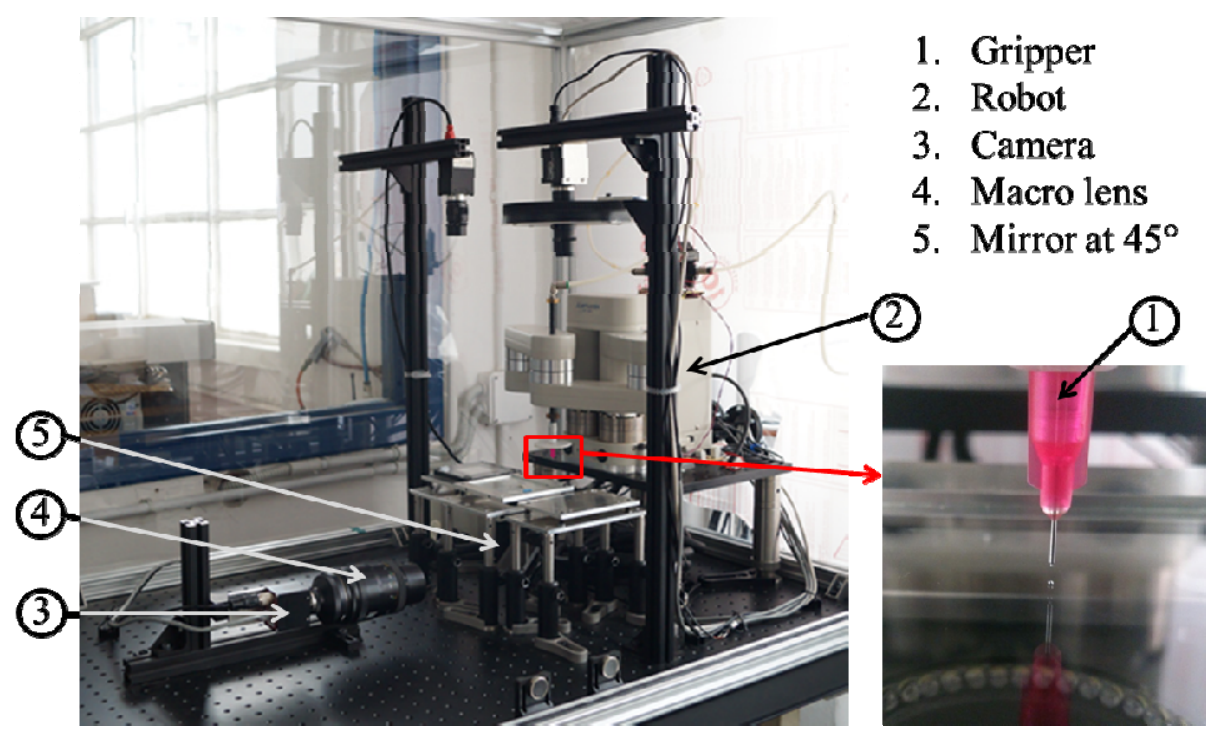

Fig. 1. The micromanipulation workcell and the innovative gripper with release needle 
The gripping performance was evaluated comparing the measured position of the barycenter and the orientation of the component in two images acquired before and just after the gripping respectively. Similarly, the release was evaluated by detecting and measuring its pose before and after the release.

With the same approach adopted in [6], the performance indices we calculated for each test cycle were the repeatability and accuracy, according to the definition given by the international standard ISO 9283. Moreover, the gripping and release percentages, representing the success of the correspondent operations, were calculated.

As introduced above, in previous studies we demonstrated that the use of the positive pressure, in practice a soft blow, represented an acceptable method to assist the microcomponent release.

For this reason, the first class of experiments we executed considered the use a standard vacuum gripper, grasping the component by simple activation of the vacuum generation system, but releasing it by providing a blow for few milliseconds. A set of pick and place cycles was performed. While the gripping of the ball was always successful (gripping percentage of 100\%), the release phase highlighted a strong dependence of the release performance on the blow pressure. Therefore two sets of experiments were carried out varying the blow pressure, the former trying to obtain an accurate and repeatable release independently from the release percentage, the latter trying to maximize the release percentage. The results for repeatability and accuracy for the first condition are graphically reported in Fig. 2, those related to the second condition are reported in Fig. 3. The origin of the reference system in the graphs represented the target position, while the small crosses indicated the barycenter of the ball for each cycle.

Gripping Test

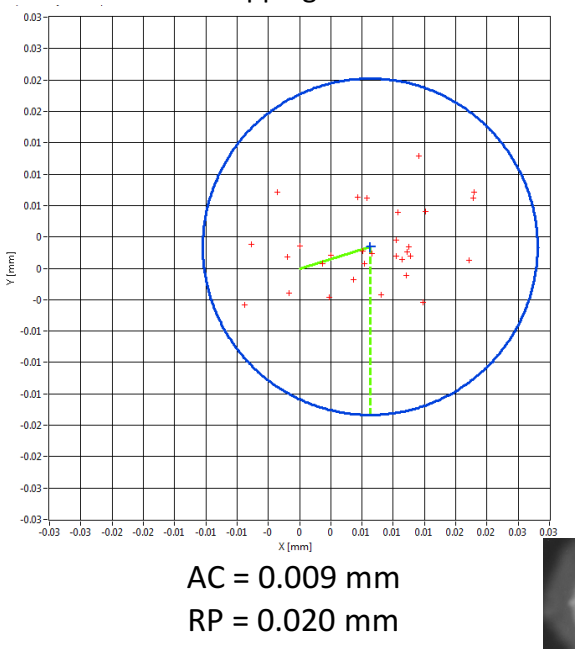

Release Test

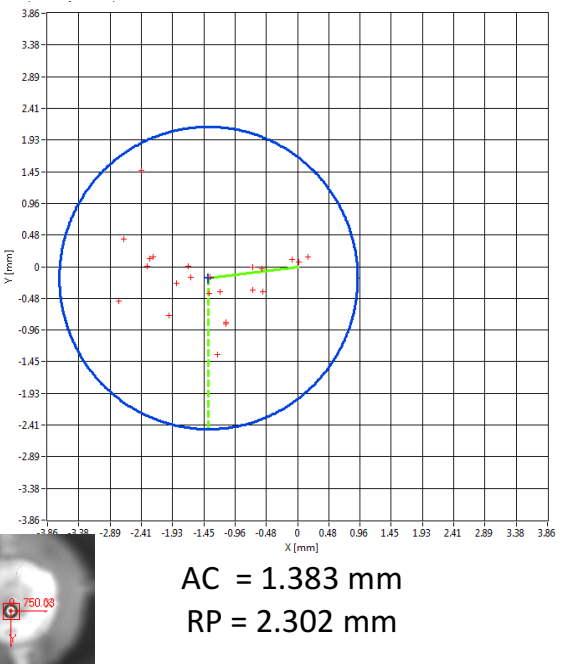

Fig. 2. Results for standard vacuum gripper manipulating the ball: gripping percentage of $100 \%$ and release percentage of $82.14 \%$ 
Gripping Test

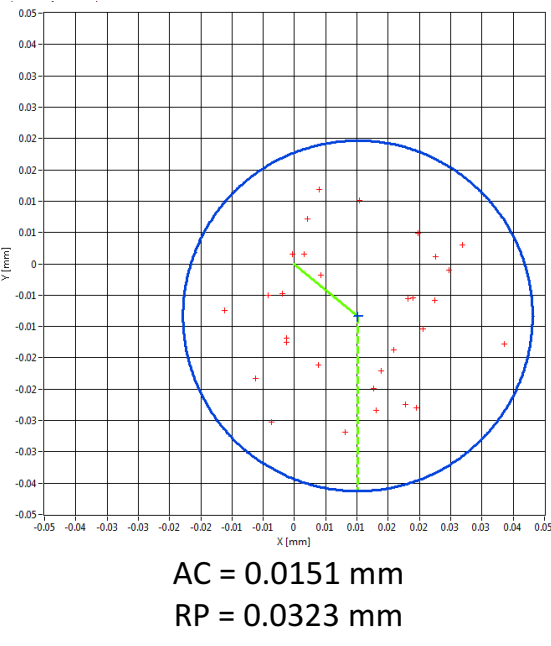

Release Test

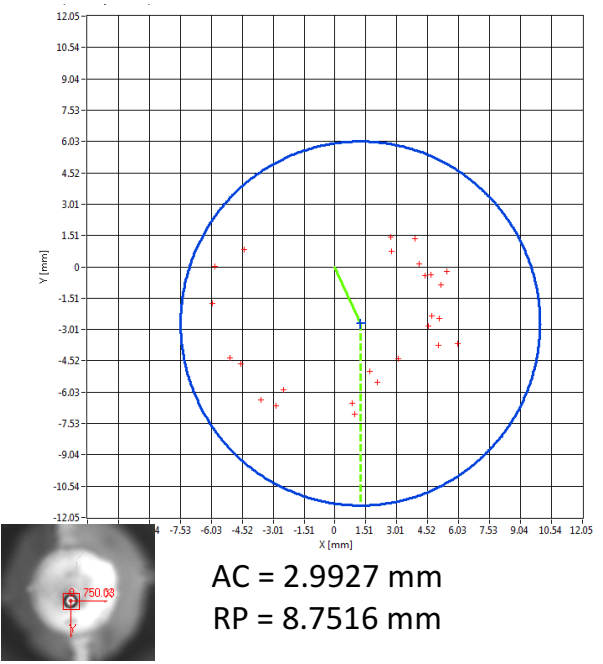

Fig. 3. Results for the standard vacuum gripper manipulating the ball: gripping percentage of $100 \%$ and release percentage of $86.67 \%$

The experiments were repeated with the resistance and the results are shown in Fig. 4. Opposite to the ball, the resistance could have various orientations, therefore the accuracy and the repeatability values of the gripping and the release operations have been calculated to evaluate the orientation error and reported in Table 1.

As shown in Fig. 2-3, the percentage of release is not $100 \%$ while the accuracy and repeatability values are sometimes too high for ultra-precise manipulation. This is evident in Table 1, showing high values for both the orientation accuracy and repeatability.

The results in Fig. 4 also shows values of the gripping accuracy and repeatability for the resistance higher than those for the solder ball, most likely due to a sort of auto-centering property of the ball on the gripper tip. Moreover, the tuning of the pressure parameters can be onerous and the performance indices very sensitive to small changes of their values.

The innovative microgripper has been designed to combine the advantages of the vacuum gripping method and overcome all the difficulties related to the use of positive pressure, since no external actuation is needed to assist this phase. To investigate this gripper and the manipulation method, we repeated the experiments on the solder ball.

The gripping and release accuracy and repeatability are reported in Fig. 5. In this case, the performance indices for the release are much better than those obtained with the standard gripper and the positive pressure assisting the release. Note also that, in both cases, the percentage of success of the gripping and release operations was $100 \%$, validating the expected effectiveness of this method. 
Table 1. Orientation accuracy and repeatability for the standard microgripper manipulating the resistance

\begin{tabular}{|c|c|c|}
\hline $\begin{array}{c}\text { Resistance: values for } \\
\text { orientation }\end{array}$ & Gripping & $\begin{array}{c}\text { Release (by positive } \\
\text { pressure) }\end{array}$ \\
\hline Accuracy $\left[{ }^{\circ}\right]$ & -0.550 & -8.331 \\
\hline Repeatability $\left[{ }^{\circ}\right]$ & 17.293 & 43.322 \\
\hline
\end{tabular}

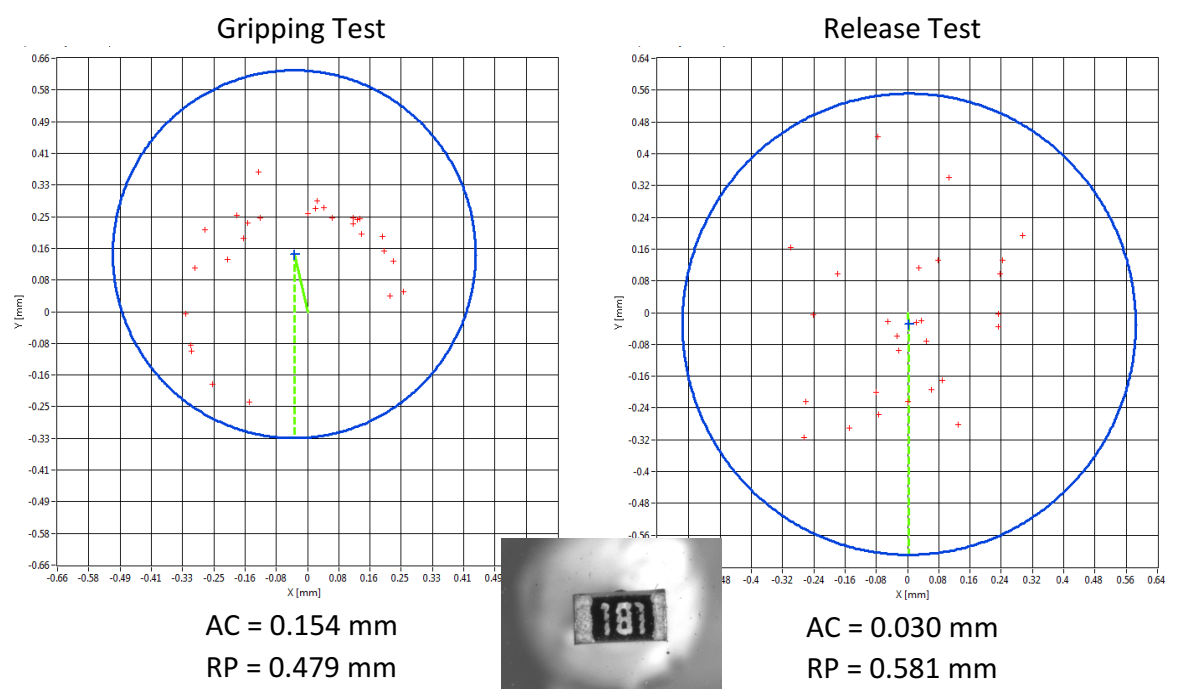

Fig. 4. Results for the standard vacuum gripper manipulating the resistance: gripping and release percentage of $100 \%$

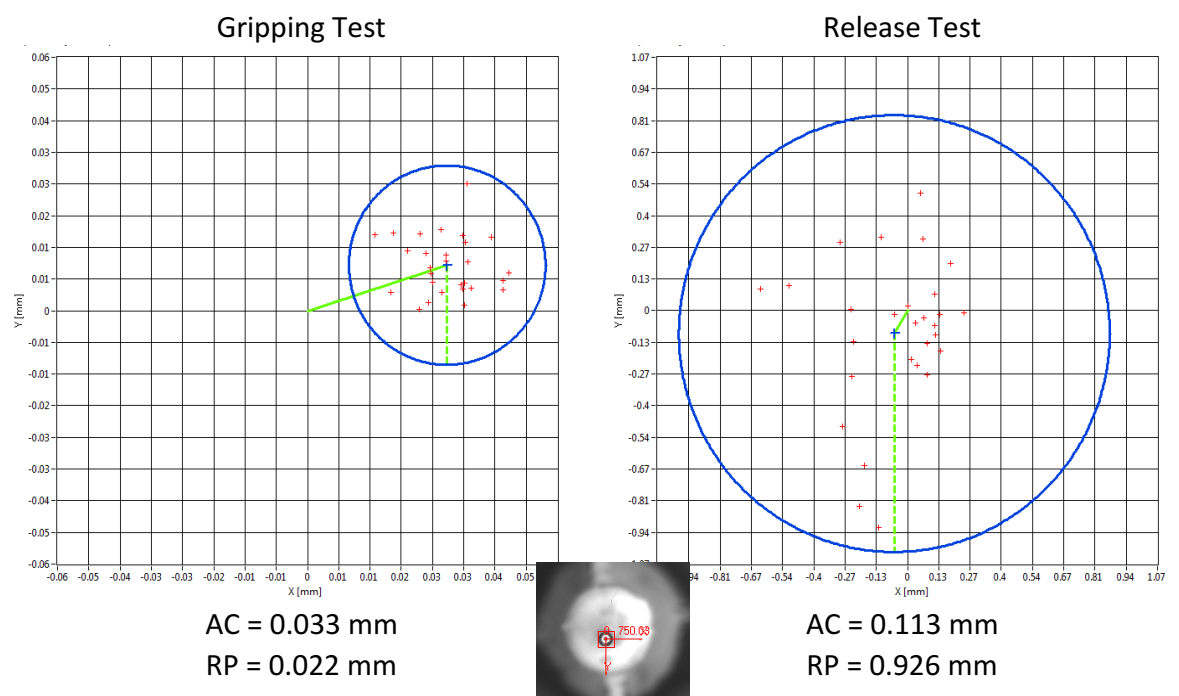

Fig. 5. Results for the innovative vacuum gripper manipulating the solder ball: gripping and release percentage of $100 \%$ 
The experiments were then repeated with the resistance and the results are shown in Fig. 6. Note that the performance of the two microgrippers in terms of both precision and reliability are comparable. As for the case of the standard microgripper, the accuracy and the repeatability values of the gripping and the release operations have been calculated to evaluate also the orientation error (Table 2).

Table 2. Orientation accuracy and repeatability for the innovative microgripper manipulating the resistance

\begin{tabular}{|c|c|c|}
\hline $\begin{array}{c}\text { Resistance: values for } \\
\text { orientation }\end{array}$ & Gripping & Release \\
\hline Accuracy $\left[{ }^{\circ}{ }\right]$ & 0.792 & 2.478 \\
\hline Repeatability $\left[{ }^{\circ}\right]$ & 10.498 & 45.759 \\
\hline
\end{tabular}

Gripping Test

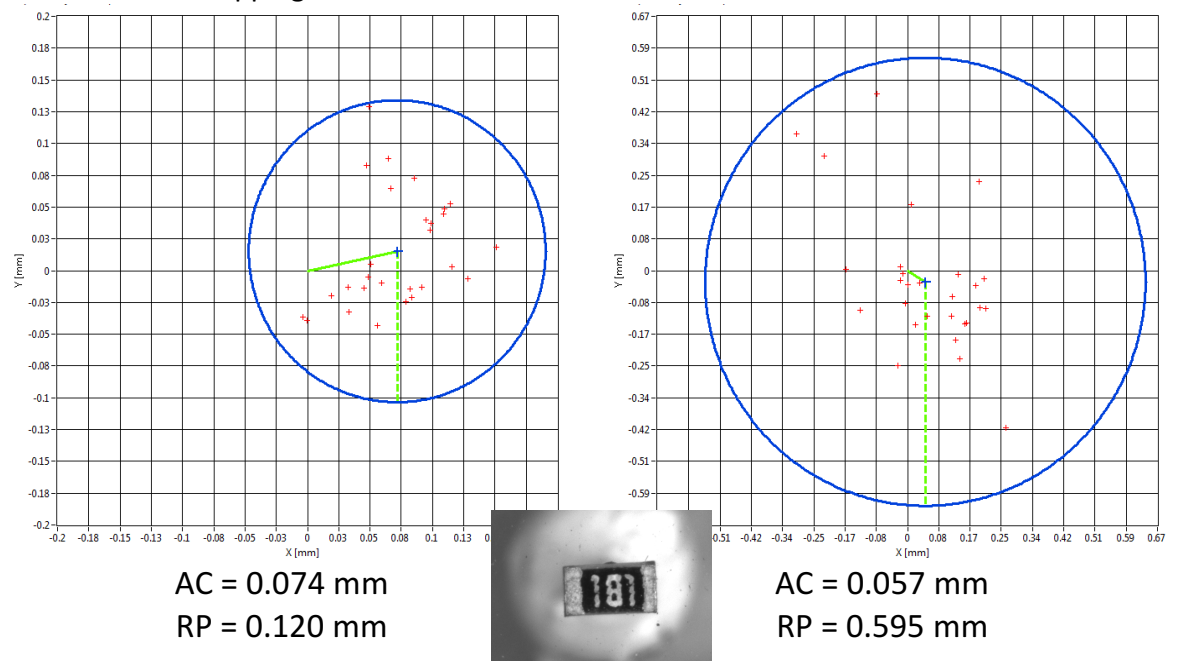

Fig. 6. Results for the innovative vacuum gripper manipulating the resistance: gripping and release percentage of $100 \%$

\section{Conclusions}

Considering the current need for more flexible and cost efficient automatic PCBs rework stations to move towards the actual implementation of the de-manufacturing paradigm, the present work devoted to the study of handling devices for the re-balling of BGA packages and PCBs and the replacement of different surface-mount components (SMCs) on the PCBs.

An innovative device and method to manipulate by vacuum a microcomponent has been presented and compared with a conventional vacuum microgripper in the execution of grasping and releasing tests of electronic components. In particular, the release of the microcomponents, often prevented or uncertain with traditional handling devices 
due to the presence of adhesive forces, has been investigated. The results demonstrated that the innovative microgripper is able to manipulate submillimetric components better than the conventional vacuum microgripper and that the special releasing system allowed a reliable and safe release. However, the values of orientation accuracy and repeatability are still high for ultra-precise manipulation, therefore additional expedients should be considered. Moreover, it can be noticed that, for both microgrippers, the repeatability values in the gripping and release phases are much higher than the accuracy values. For this reason, further efforts will be required to better comprehend this phenomenon and try to improve the grippers' performance.

Concluding, the innovative device represents an interesting solution for handling electronic components for PCBs rework and, more in general, for micromanipulation and assembly of different microproducts.

In the next future, the handling of other microcomponents with this gripper will be also tested and it will investigated more deeply in order to highlight advantages and limitations.

Aknowledgements. This research has been partially funded by Regione Lombardia, in the framework of the Accordo Quadro RL-CNR, project 'FIDEAS: Fabbrica Intelligente per la Deproduzione Avanzata e Sostenibile'.

\section{References}

1. Errington, M., Childe, S.: A business process model of inspection in remanufacturing. Journal of Remanufacturing 3, 1-22 (2013)

2. Brusaferri, A., Colledani, M., Copani, G., Pedrocchi, N., Sacco, M., Tolio, T.: Integrated De-manufacturing systems as new approach to End-of-Life management of mechatronic devices. In: 10th Global Conference on Sustainable Manufacturing, pp. 332-339 (2012)

3. Solderquik, http://www. solderquik.com

4. Ruggeri, S., Fontana, G., Fassi, I., Legnani, G., Pagano, C.: Dispositivo di manipolazione e metodo per manipolare a vuoto un componente. Italian Patent pending No. MI2013A000541 (2013)

5. Chen, T., Sun, L., Chen, L., Rong, W., Li, X.: A hybrid-type electrostatically driven microgripper with an integrated vacuum tool. Sensors and Actuators A: Physical 158(2), 320-327 (2010)

6. Ruggeri, S., Fontana, G., Pagano, C., Fassi, I., Legnani, G.: Handling and Manipulation of Microcomponents: Work-Cell Design and Preliminary Experiments. In: Ratchev, S. (ed.) Precision Assembly Technologies and Systems. IFIP AICT, vol. 371, pp. 65-72. Springer, Heidelberg (2012)

7. Fontana, G., Ruggeri, S., Fassi, I., Legnani, G.: Flexible vision based control for microfactories. In: Proc. of the 7th International Conference on Micro- and Nanosystems IDETC/MNS 2013, Portland, OR, USA, August 4-7 (2013) 